\section{A NOVEL REPRESENTATION OF ECG BEAT-TO-BEAT} VARIATION

${ }^{1}$ Nan Liu*, ${ }^{2}$ Dagang Guo, ${ }^{2}$ Zhi Xiong Koh, ${ }^{2}$ Andrew Fu Wah Ho, ${ }^{1,2}$ Marcus Eng Hock Ong. ${ }^{1}$ Duke-NUS Medical School, National University of Singapore, Singapore; ${ }^{2}$ Department of Emergency Medicine, Singapore General Hospital, Singapore

\subsection{6/bmjopen-2019-EMS.14}

Background Heart rate variability (HRV) is believed to strongly associate with autonomic nervous system. So far, majority of efforts in HRV research are deriving sophisticated parameters with linear and nonlinear techniques. Furthermore, researchers have been focusing on developing advanced signal processing tools for efficient noise removal and accurate QRS detection, prior to HRV parameter calculation.

Method We propose a novel representation of beat-to-beat variation in ECG, called heart rate n-variability $(\mathrm{HRnV})$, as an alternative to conventional HRV. The derivation of $\mathrm{HRnV}$ parameters are based on $\mathrm{n} R \mathrm{R}$ intervals with or without overlaps. We can create many sets of $\mathrm{HRnV}$ parameters which are promising at generating extra information from limited data source. We conducted a simulation study by using the ECG record of subject \#16 265 from MIT-BIH Normal Sinus Rhythm Database.

Results Among the time domain parameters, we observed that the values were generally incremental with the increase of $n$. We observed the same trend of value change in frequency domain parameters. In nonlinear analysis, the differences between HRV and HRnV on Poincare plot measures were obvious, while those on entropy and detrended fluctuation analysis (DFA) metrics were not.

Conclusion HRnV measures enable us to augment the conventional HRV with many more parameters. We believe that $\mathrm{HRnV}$ is an important addition to HRV and will have great potential in analyzing prehospital ECGs.

Conflict of interest None.

Funding SingHealth Foundation Research Grant SHF/FG652P/ 2017.

\section{CARDIAC ARREST SURVIVAL VERSUS DEFIBRILLATION DELAY DURING ONGOING CPR}

O Franěk* , J Pekara. EMS City of Prague, Czech Republic

\subsection{6/bmjopen-2019-EMS.15}

Background In case of cardiac arrest with ventricular fibrillation (VF CA), several studies confirmed decrease of survival chance by $10 \%$ per minute when resuscitation is NOT performed. On the other side, only a rare data are available to evaluate the influence of defibrillation delay when resuscitation IS performed until defibrillation is available.

Method This is a retrospective analysis of VF CA from Prague Utstein-style registry data from 2003 to 2018. We compared the outcome of patients where the collapse due to ventricular fibrillation onset was directly witnessed by EMS crew so that the defibrillation was available immediately (EMS-WITNESSED group) with outcome of patients who collapsed before emergency call, layperson CPR (without AED) was provided and the patients were found with VF CA by responding EMS crew (LAYPERSON-WITNESSED group).

Results In EMS-WITNESSED group there were 325 patients and 151 survivors (CPC 1-2), while in LAYPERSON-WITNESSED group there were 1741 patients and 679 survivors.
That gives survival rates $46.5 \%$ and $39.0 \%$ respectively; $\mathrm{RR}=0.88$. The average EMS response time (call-to-patient) was $8: 48 \mathrm{~min}$.

Conclusion If layperson CPR is performed from collapse to EMS arrival, the chance of survival from VF CA with defibrillation after almost $9 \mathrm{~min}$ after collapse reached as much as $88 \%$ as compared to the ideal situation where immediate defibrillation is available. Thus, maximal effort including dispatcher-assisted CPR should be focused to support layperson CPR. More studies are needed to clearly evaluate the real life benefit of AED programs in municipal setting.

Conflict of interest None.

Funding None.

\section{LONG TERM EFFECTS OF DISPATCHER-ASSISTED CPR - DID WE TOUCH THE CEILING?}

O Franěk* , J Pekara, P Sukupova, M Pohlova. EMS City of Prague, Czech Republic

10.1136/bmjopen-2019-EMS.16

Background Dispatcher-assisted CPR (D-CPR) is recognized to be an important part of the 'chain of survival' in case of preEMS-arrival out-of-hospital cardiac arrest (OHCA). In Prague, D-CPR was introduced in 2003 and since 2006 it should be provided in a routine manner for all calls suspected from OHCA. The aim of the study is to compare the influence of $\mathrm{D}-\mathrm{CPR}$ to bystander CPR (B-CPR) rates for confirmed OHCA cases in different phases of D-CPR implementation.

Method This is a retrospective analysis of B-CPR rates from Prague Utstein-style OHCA registry after 5, 10 and 15 years of implementation of D-CPR, as compared to the 'baseline' in 2003.

Results Rates of B-CPR in 2003, 2008, 2013 and 2018 were $13.6 \%, 69.2 \%, 81.6 \%$ and $81.0 \%$ respectively. There was a highly significant difference between 2003 and 2008 rates $(\mathrm{p}<0,0001)$, as well as between 2008 and 2013 rates $(\mathrm{p}<0,01)$, but there is no difference between 2013 and 2018 rates $(\mathrm{n} / \mathrm{s})$.

Conclusion Full implementation of D-CPR in Prague took more than 5 years. The implementation was associated with very significant increase of B-CPR ratio, which reached as much as $80 \%$ in the second period $(5-10$ years after start of the implementation). Nevertheless, after reaching this level, the L-CPR ratio now remains stable for several years and it seems this method reached its maximum. To increase B-CPR rates significantly over $80 \%$ we probably need some new approaches.

Conflict of interest None.

Funding None.

\section{HOW FAR AND HOW LONG TO DO? PCO2 AND LACTATE AS POSSIBLE PREDICTORS OF SURVIVAL IN TRAUMATIC CARDIAC ARREST}

C Camacho*, G Mancho, R De Elias, Y Lahoz, E Corral. SAMUR Protección Civil, Madrid, Spain

\subsection{6/bmjopen-2019-EMS.17}

Background The factors associated with the survival of Traumatic cardiac arrest (TCA) have been analyzed by many authors. Trying to define a limit on resuscitation efforts: How 